\begin{tabular}{|c|c|c|}
\hline $\begin{array}{l}|N F G A| N \\
P \cup B L \mid C A T I O N\end{array}$ & $\begin{array}{l}\text { International Journal of Advanced Engineering, Management and Science } \\
\text { (IJAEMS) } \\
\text { ISSN: 2454-1311 } \\
\text { Vol-7, Issue-3; Mar, } 2021 \\
\text { Journal Home Page Available: } \underline{\text { https://ijaems.com/ }} \\
\text { Journal DOI: https://dx.doi.org/10.22161/ijaems } \\
\text { Article DOI: } \text { https://dx.doi.org/10.22161/ijaems.73.6 }\end{array}$ & 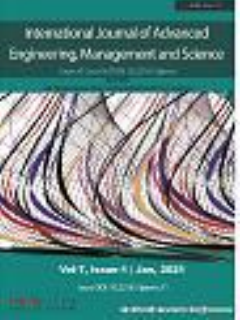 \\
\hline
\end{tabular}

\title{
Fake News Detection using Machine Learning: A Review
}

\author{
Priyanshi Goyal ${ }^{1}$, Dr. Swapnesh Taterh ${ }^{2}$, Mr. Ankit Saxena ${ }^{3}$
}

${ }^{1}$ Student, Amity University Rajasthan, India

${ }^{2}$ Professor, Amity University Rajasthan, India

${ }^{3}$ Assistant Professor, Department of CSE, Invertis University, Bareilly, India

Received: 28 Nov 2020; Received in revised form: 27 Jan 2021; Accepted: 15 Feb 2021; Available online: 15 Mar 2021

(C)2021 The Author(s). Published by Infogain Publication. This is an open access article under the CC BY license

(https://creativecommons.org/licenses/by/4.0/).

\begin{abstract}
This paper examines the implementation of natural Techniques of language recognition for 'false news' identification, that is, false news storeys that stem from unreputable storeys from sources. Using a data set and list obtained from Signal Media for OpenSources.co sources, we use the expression frequency-inverse-inverse Detection of bi-grams and probabilistic meaning free grammar (PCFG) document frequency (TF-IDF) in a corpus of articles.[1] Fast Access and Exponential Growth Social networking network data has been made available. It is difficult to analyze between false and true facts. The simple dissemination of data by sharing has contributed to a rapid rise in its falsifying. The credibility of social media networks is also at stake if there is a proliferation of the dissemination of false information. It has now become a study activity to check the data automatically so that it is classified as false or accurate by its source, content and publisher. Machine learning, along with some pitfalls, has played a critical role in the classification of results. This paper explores various approaches to machine learning to distinguish fake and fabricated news. The restriction of such methods and improvisation by the use of deep learning is also explored. [2]
\end{abstract}

Keywords - Machine learning, Classification algorithms, Fake-news detection, Text classification, online social network security, social network.

\section{INTRODUCTION}

Fake news is now seen as one of the major problems of democracy, Journalism, the economy, guy. It has weakened the general confidence in the government and has a potential influence on life today. [3] The notion of misleading news is not a revolutionary one. Notably, even before the invention of the Internet, the idea existed when newspapers used imprecise and distorted information to promote their purposes. More and more consumers have continued to forsake traditional media channels used to disseminate data on Internet networks through the introduction of the Internet. Not only does the above approach encourage users to browse a variety of publications in one session, it is is more usable and faster. However, the development came with a redefined notion of fake news as content publishers began to use what was commonly referred to as click bait. Click baits are phrases that are intended to capture the attention of a customer who is brought to a web page whose content is significantly below their expectations by clicking on a link. Many users find clickbaits to be an annoyance, and the result is that most of these tourists will only end up visiting certain sites for a very short time.[4] A few decades ago, the term "Fake News" was much less unheard of and not popular, but it has exploded as a big monster in this digital era of social media. In our society, fake reporting, clouds of knowledge, manipulation of news and loss of confidence in the media are increasing problems. However, an in-depth understanding of false news and its origins is required in order to begin to address this problem. Only then can we look at the different strategies and fields of machine learning ( ML), natural language processing (NLP) and artificial intelligence ( $\mathrm{AI}$ ) that might enable us to resolve this situation. In the last half- 
year, "fake news" has been used in a multitude of ways and various interpretations have been given.[5] A considerable number of pre-existing false news models are context-specific in nature. The mechanism to identify the categories of disappointments that may arise in the handling of textual material is missing. This paper explores a variety of strategies and kinds of dissatisfaction that can be faced in managing online news and measures their benefits and advantages. Mathematical formulas inconvenience. The solution of the problem in question offers an algorithmic approach. The article discusses the following features of fake news in order to discriminate between the different current models:[10]

(a) Describes the content, forms and features of fake news.

(b) false news outlets are detected.

(c) an overview of the different entities (data collections) which can be used for classifying false news.

(d) Developing a data model to identify the related news information

(e) Evidential retrieval, setting up false news criteria.

(f) for the purposes of predicting the classification, control, collection and use of data.[10]

\section{OUTLINE}

Text, or natural language, is a type that is difficult to process due to different linguistic characteristics and forms, such as sarcasm, metaphors, etc. In addition, thousands of languages are spoken and each language has its own grammar, script and syntax. The processing of natural language is a branch of artificial intelligence that involves techniques that can use text, create models and make predictions. The aim of this work is to establish a system or model that can use data from past news reports to assess whether or not a news store is likely to be false.[5]

\subsection{MOTIVATION:}

Fake news spreads mainly across social networking networks such as Facebook, Twitter and many others. In order to hurt a person, and/or benefit financially or politically, fake news is written and released with the intent to deceive. Currently, the vertical litany spanning national security, education and social media is seeking to find better ways to tag and describe misleading news in order to defend the public from disinformation. Our goal is to create a clear model that classifies the news store as either inaccurate or true. Following media attention, Facebook has recently been at the forefront of much criticism. They have now released a tool to review false news on the website itself for their users, and it is apparent from their recent announcements that they are actively researching their ability to automatically recognize those tweets. It is not, however, a clear task. As fake news exists at all ends of the spectrum, the algorithm can be ideologically impartial to offer an equal balance of reputable news sources at either end of the spectrum. We should decide what makes it 'legitimate' for a digital medium and an empirical instrument to evaluate this.[8]

\subsection{CLEANING TEXT DATA:}

Data cleaning has been carried out at different stages in this process. Next the data was checked for null values and redundant columns, and as there were columns that did not add value to the project, they were discarded. The next step was to delete the stop words from the results. The explanation for the deletion of stop words is that the model causes dimensionality. Elimination of the stop terms will also further limit the dimensionality of the model. The WordNetLemmatiser package was then used to lemmatize the data. Lemmating is a means of replacing words with general sense, e.g. buy, supermarket, store. Only the word "Store" can be omitted from the other two words if the lemma is ended. In this way, they will not be taken as three distinct words when the text matrix is created, thereby reducing time and complexity. Finally, by converting data into lower cases the data is unified. This is the key step, since the duplication of the data can be reduced.[9] 


\section{METHODOLOGY}

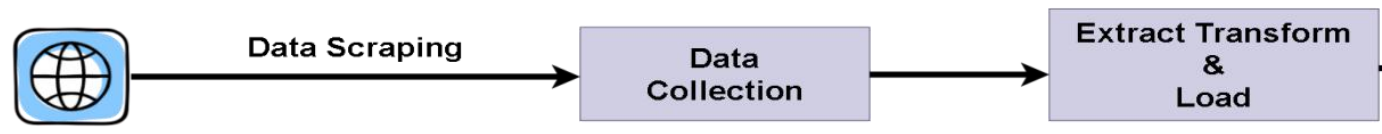

World Wide Web

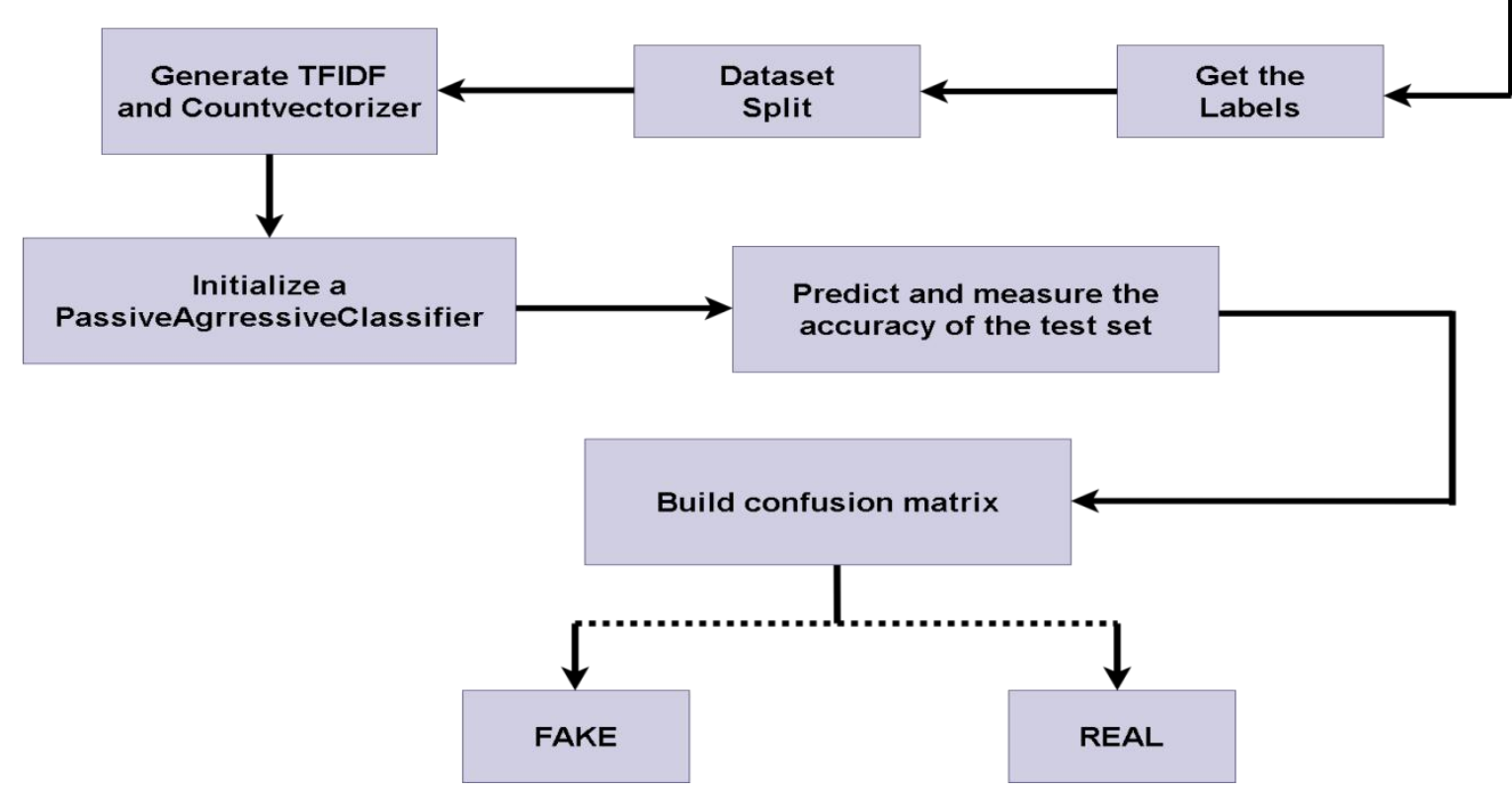

Fig.1: Classifier prediction model

Depending on the size and consistency of the text data (or corpus) and also the characteristics of the text vectors, the output of the classifier can differ. As it comes to extracting text attributes, the usual noisy terms called 'stop words' are less relevant words, they do not add to the true sense of the expression and they only contribute to the dimensionality of the function and can be omitted for better performance.[5] This helps to minimize the size / dimensionality of the text corpus and apply text history to isolate the function. Lemmatization is also used to transform terms into their central context, resulting in the conversion of several words into a single, distinct representation.

\section{MODEL}

The detail is never evenly distributed in the data collection. In such cases, however, the performance of the classifier may be calculated. The accurate predictions of the classifier are truth positive, and the incorrect predictions are false positive. The role of calculating precision, recall and $\mathrm{f} 1$ scores is made straightforward by the use of these figures.

\begin{tabular}{|c|c|c|c|c|}
\hline \multicolumn{2}{|c|}{} & \multicolumn{3}{|c|}{ Forecast Class } \\
\cline { 3 - 5 } & \multicolumn{3}{|c|}{ Classified Positive/Negative } \\
\hline \multirow{2}{*}{ Real Class } & $\begin{array}{c}\text { Labeled } \\
\text { Positive/Negative }\end{array}$ & Class = Real & Class = Fake \\
\cline { 3 - 5 } & & & True Positive & False Negative \\
\cline { 3 - 5 } & & Class = Fake & False Positive & True Negative \\
\hline
\end{tabular}

Fig 2: Confusion matrix model 


\begin{tabular}{|c|c|c|}
\hline $\begin{array}{l}\text { INFMGAIN } \\
P \cup B L I C A T I O N\end{array}$ & $\begin{array}{l}\text { International Journal of Advanced Engineering, Management and Science } \\
\text { (IJAEMS) } \\
\text { ISSN: 2454-1311 } \\
\text { Vol-7, Issue-3; Mar, } 2021 \\
\text { Journal Home Page Available: } \underline{\text { https://ijaems.com/ }} \\
\text { Journal DOI: https://dx.doi.org/10.22161/ijaems } \\
\text { Article DOI: } \text { https://dx.doi.org/10.22161/ijaems.73.6 }\end{array}$ & 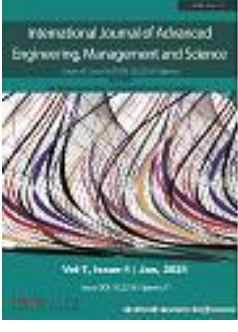 \\
\hline
\end{tabular}

\section{FAKE NEWS CLASSIFICATION:}

The various forms of fake news of this paper are summarized below in their latest paper.

1. Visual based: Visual fake news uses content that incorporates multiple media forms including graphic display such as Photo-shopped images and videos. Visual news is mainly available on platforms such as Social Media and Media websites, attracting the attention of the public. For many other users, Facebook, Instagram and Twitter are common examples of social media used to publish and share content online.

2. User based: Fake accounts produce this kind of fabricated news and reach particular demographics that could reflect those age groups, ethnicity, community, political affiliations.[6]

3. Fake headlines: Headlines for attracting publicity that represent fictitious reality. They are also used for less credible journals, such as tabloid newspapers. Readers also quickly note that the content of the storey does not match the headline. Their names are referred to as "Clickbait Headlines."

4. Target misinformation: Fictitious piece of information shared for self-serving purposes. Targeted disinformation is frequently aimed at audiences most vulnerable to obtaining this sort of material without checking its validity and quickly embracing and distributing polarizing news.

\section{COMPARISON}

A main aspect of the grouping of findings is the correlations between intra-class and inter-class clusters. The cluster intra-class indicates the distance between the data point and the cluster centre, while the cluster between the cluster and the data point displays the distance between the cluster.The distance between the cluster data point and the cluster data point.

Various characteristics were selected for performance observation using the various methods of supervision and deep learning mentioned above. There are essentially four attribute vectors derived from our text dataset.

\footnotetext{
* Vector number *

* Phrase-level vectors

* $\mathrm{N}$-gram vectors

* Vectors of character type[7]
}

\section{LITERATURE SURVEY}

\subsection{PREVIOUSLY USED TECHNIQUES:}

Social media may also act as an inconsistent platform for false news and inaccurate facts, a popular source of news for newspapers and TV. According to recent estimates, Facebook has 1,2 billion users on the most popular social media site. Thus, blogs such as this are certainly one way in which many people share counterfeit news widely. But to find misleading news on social media sites is very difficult. Psychological and social theories for appraisal from a data review point of view should be considered. The reasons for reading news on these websites can differ. Few will take less time, share and comment on the topic of the post, debate on the issue, etc. There are a few steps to take, from characterizing these news outlets to recognizing them.[10]

\subsection{SOME FREQUENTLY OCCURRING FAKE NEWS FORMS:}

It is important to recognize the same thing and to observe the various types that may constitute it before dwelling on the topic of false news. Fake news is a type of sensational reporting or purposeful advertising that includes the propagation of intentional disinformation or hoax by conventional print, communicative news media or online social media. Periodically, the news is however, sometimes it also finds its way into the mass press through the deceit of social media. Fake news is published and disseminated strategically with the goal of deluding or destroying an office, a substance, a person or raising money through frequently leveraging nostalgic or deceptive features with a relentless effort to expand consumer flow.[10]

\section{RESULT}

Our research started with the extraction of real-time tweets using keywords, and after the pre-processing of these tweets, important features were extracted from the dataset. These characteristics are important because they have valuable features that define the data collection.

We research the predictive consistency and device variability. We rely only on higher performance models for the assessment of models in terms of coherence and heterogeneity. We cluster the model space and carry out an 
inquiry to explain the function of the characteristics of the model choices depending on the characteristics present in every model[9].By analyzing all the templates used to accomplish the purpose, we calculate the functionality's predictive precision. More precisely than the average AUC values of all models in which the feature was used, is predictive precision of the function. Similarly, the system variability is the Insane average value of all the models used by the function. How functions are achieved is mathematical precision and ambiguity. A few features obviously exhibit a significantly higher precision in the measurement.[9].It is also clear how much precision and quantity of training results are affected by the false news identification paradigm. If the model is trained with a complex data set with news from various domains, it is not too far-reaching to achieve a much more stable and reliable classification. More technological innovations, including hyperparameter tuning and improved feature range, can also be used in this guide.[5]

\section{CONCLUSION}

In recent years the issue of fake news and its impact on culture has been highly concerned. In the issue of false news identification, the subject of data prediction and classification should been controlled using training data. Since most falsified news databases have many features, most are useless and obsolete, decreasing the amount of falsified news detection algorithm can improve its accuracy. Therefore a method of false news identification should be used in this article to gather features. The key characteristics in the function selection system are clustered into separate clusters, depending on the comparability of the characteristics. From each cluster, the final feature set is then selected depending on the necessary characteristics. [12] Finally, our results suggest that models with odd combinations of features appear to recognise these kinds of false news. As a result different models are based on a very different logic, distinguishing false stores from real ones. This shows the scale of the problem and helps us to understand how impossible it is for a single approach to fix all kinds of false news reports. We expect fake news stores to be classified as a technique for creating solid and accurate classifier sets as a potential task. For example, we've seen a number of cluster models that are made up of random variations of features in this work. This means that the Ensemble Integrating Models strategies from different clusters are in place. This is a fruitful line of inquiry.[10] Fake news has been steadily detected in recent years. However an item of news has also been found to be false. In our study, Explanatory False News Identification is a novel challenge, which seeks to:
1) dramatically boost detection efficiency; and 2) use news phrase describing why news stores are deemed false; and customer knowledge. In order to research counterfacts and to detect causal statements/comments, we suggest a strong hierarchical joint attention network. Real-world data set tests show the feasibility of the proposed system.[13]

\section{REFERENCES}

[1] Gilda, S. (2017, December). Evaluating machine learning algorithms for fake news detection. In 2017 IEEE 15th Student Conference on Research and Development (SCOReD) (pp. 110-115). IEEE.

[2] Manzoor, S. I., \& Singla, J. (2019, April). Fake News Detection Using Machine Learning approaches: A systematic Review. In 2019 3rd International Conference on Trends in Electronics and Informatics (ICOEI) (pp. 230234). IEEE.

[3] Zhou, X., Zafarani, R., Shu, K., \& Liu, H. (2019, January). Fake news: Fundamental theories, detection strategies and challenges. In Proceedings of the twelfth ACM international conference on web search and data mining (pp. 836-837).

[4] Aldwairi, M., \& Alwahedi, A. (2018). Detecting fake news in social media networks. Procedia Computer Science, 141, 215-222.

[5] Agarwal, V., Sultana, H. P., Malhotra, S., \& Sarkar, A. (2019). Analysis of Classifiers for Fake News Detection. Procedia Computer Science, 165, 377-383.

[6] Manzoor, S. I., \& Singla, J. (2019, April). Fake News Detection Using Machine Learning approaches: A systematic Review. In 2019 3rd International Conference on Trends in Electronics and Informatics (ICOEI) (pp. 230234). IEEE.

[7] Mahir, E. M., Akhter, S., \& Huq, M. R. (2019, June). Detecting Fake News using Machine Learning and Deep Learning Algorithms. In 2019 7th International Conference on Smart Computing \& Communications (ICSCC) (pp. 15). IEEE.

[8] Sharma, N., 2020. Fake News Detection using Machine Learning. Open Access, [online] 4(4), pp.1317-1320. Available at: <https://www.ijtsrd.com/computerscience/other/31148/fake-news-detection-using-machinelearning/nikhil-sharma> [Accessed 12 June 2020].

[9] Haridas, N. (2019). Detecting the Spread of Online Fake News using Natural Language Processing and Boosting Technique (Doctoral dissertation, Dublin, National College of Ireland).

[10] Reis, J. C., Correia, A., Murai, F., Veloso, A., \& Benevenuto, F. (2019, June). Explainable machine learning for fake news detection. In Proceedings of the 10th ACM Conference on Web Science (pp. 17-26).

[11] Ahmad, F., \& Lokeshkumar, R. A Comparison of Machine Learning Algorithms in Fake News Detection.

[12] Yazdi, K. M., Yazdi, A. M., Khodayi, S., Hou, J., Zhou, W., \& Saedy, S. (2020). Improving Fake News Detection Using K-means and Support Vector Machine 
Approaches. International Journal of Electronics and Communication Engineering, 14(2), 38-42.

[13] Shu, K., Cui, L., Wang, S., Lee, D., \& Liu, H. (2019, July). defend: Explainable fake news detection. In Proceedings of the 25th ACM SIGKDD International Conference on Knowledge Discovery \& Data Mining (pp. 395-405). 